\title{
Content changes in TGF- $\beta$ and IGF-1 and their significance in the discs of diabetic rats
}

\author{
Ji-long $\mathrm{An}^{\mathrm{a}, \#}$, Ya-peng Wang ${ }^{\mathrm{b}, \#}$,Ya-peng Sun ${ }^{\mathrm{c}}$ \\ ${ }^{a}$ Department of orthopedics, Affiliated Hospital of Hebei University. Baoding, 071000, China. \\ ${ }^{b}$ Orthopaedics Department, Wuxi Ninth Pepole's Hospital Affiliated to Soochow University, Wuxi ,214000, China. \\ ${ }^{C}$ Department of Spine Surgery, The Third Hospital of Hebei Medical University, Shijiazhuang, 050000, China.
}

\begin{abstract}
Objectives: To measure the content changes in transforming growth factor- $\beta$ (TGF- $\beta$ ) and insulin-like growth factor-1 (IGF-1) in discs in diabetic rats and normal rats in three different periods after treatment with streptozocin (STZ) and to discuss the significance of the content changes.

Methods: Thirty Sprague-Dawley (SD) rats were divided into the treated group and the control group, with 15 rats in each group. In the treated group, the STZ solution was injected into the peritoneal of SD rats to establish the diabetic model. In the control group, no special treatment was performed on the SD rats. Complete lumbar discs in the two groups were obtained in the second, fourth, and sixth week, respectively. After paraffin-embedded sections and hematoxylin and eosin (HE) staining, the morphology changes in the disc were observed, and the TGF- $\beta$ and IGF-1 contents were measured by the immunohistochemical method. Results: HE staining showed degenerative changes in the disc in the treated group in the three different periods, but no changes were observed in the disc in the control group. The immunohistochemical method showed that the TGF- $\beta$ and IGF- 1 contents in the treated group were significantly lower than those in the control group in the three different periods $(p<0.05)$. No significant differences were found between TGF- $\beta$ and IGF-1 in the two groups in the three different periods ( $p>0.05$ ).

Conclusion: Compared with those in the control group, the disc of STZ-induced diabetic rats in the treated group exhibited degenerative changes, and the TGF- $\beta$ and IGF- 1 contents significantly decreased. Thus, the decrease in the TGF- $\beta$ and IGF-1 contents caused by diabetes could be one of the factors causing disc degeneration.
\end{abstract}

Keywords: TGF- $\beta$, IGF-1, streptozocin, disc degeneration, diabetic rats.

\section{INTRODUCTION}

Diabetes is a disease that poses a serious threat to human health ${ }^{[1]}$. Its main characteristic is hyperglycemia and is accompanied by a series of complications. Microangiopathy is one of the main complications that cause retinopathy, diabetic nephropathy, and diabetic cardiomyopathy. It also can affect disc tissue ${ }^{[2]}$. The disc has no vascular tissue, and the nutrients diffusing into the disc rely on the marrow cavity, sinusoid, and endplate cartilage interface ${ }^{[3]}$. Diabetes-induced microangiopathy affects capillary sprouts deep into the endplates, causing morphological destruction, reduction of the number of vessels, and narrowness, which result in metabolite deposition and intervertebral disc

*Corresponding author: Zhang W,

Mailing address: Department of Spine Surgery, The Third Hospital of Hebei Medical University.

Address: 139 Ziqiang RD, Shijiazhuang 050051, People's Republic of China.

E-mail: myth_beyond@163.com

Received: 25 January 2018 Accepted: 20 March 2018 degeneration. ${ }^{[4,5]}$ Hyperglycemia diffuses into the disc and makes a concentration difference in it. It also can increase glycation end products and cause polyol pathway and endoplasmic reticulum stress ${ }^{[6]}$, which mediates the apoptosis of disc cells. The disc can be affected by some cytokines. Tumor necrosis factor (TNF) and interleukin can further promote the apoptosis of the intervertebral disc through the proinflammatory effects ${ }^{[7]}$. Transforming growth factor- $\beta$ (TGF- $\beta$ ) and insulin-like growth factor-1 (IGF-1) can regulate the synthesis of the disc extracellular matrix and stop the apoptosis of disc cells. Although TGF- $\beta$ and IGF-1 have a positive effect on the disc, whether there exists an abnormal expression in the disc of diabetic patients remains unknown. This abnormal expression is one of the factors leading to disc degeneration. In this study, diabetic rats treated by streptozocin (STZ) were chosen, and discs were obtained in the second, fourth, sixth week. Hematoxylin and eosin (HE staining) and the immunohistochemical method were performed. The TGF- $\beta$ and IGF-1 contents in the discs of diabetic rats and normal rats were measured and analyzed 
Table 1 Comparison of blood glucose concentration in rats in different periods $(x \pm S) \mathrm{mmol} / \mathrm{L}$

\begin{tabular}{lllll}
\hline $\begin{array}{l}\text { Group } \\
\text { concentration }\end{array}$ & $\begin{array}{l}\text { Initial glucose } \\
\text { concentration }\end{array}$ & $\begin{array}{l}\text { Glucose } \\
\text { concentration at } \\
\text { the second week }\end{array}$ & $\begin{array}{l}\text { Glucose } \\
\text { concentration at } \\
\text { the fourth week }\end{array}$ & Glucose \\
\hline Treated group & $24.77 \pm 5.34$ & $26.22 \pm 5.18$ & $23.76 \pm 4.46$ & $25.53 \pm 6.52$ \\
Control group & $4.94 \pm 0.65$ & $5.01 \pm 0.66$ & $4.89 \pm 0.82$ & $4.63 \pm 0.58$ \\
\hline
\end{tabular}

to determine if they were a factor causing disc degeneration.

\section{MATERIAIS AND METHODS}

Primary treatment of animal models and specimens

Animals

Table 2 Comparison of TGF- $\beta$ level in disc in control group at three different periods

\begin{tabular}{lcccc}
\hline Weeks & N & Rank mean of TGF- $\beta$ & df & p \\
\hline 2 & 5 & 7.00 & 2 & 0.368 \\
4 & 5 & 8.50 & & \\
6 & 5 & 8.50 & & \\
\hline
\end{tabular}

Table 3 Comparison of TGF- $\beta$ level in disc in treated group at three different periods

\begin{tabular}{llccc}
\hline Weeks & N & Rank mean of TGF- $\beta$ & df & p \\
\hline 2 & 5 & 6.50 & 2 & 0.311 \\
4 & 5 & 8.00 & & \\
6 & 5 & 9.50 & & \\
\hline
\end{tabular}

Table 4 Comparison of IGF-1 level in disc in control group at three different periods

\begin{tabular}{lccrc}
\hline Weeks & $\mathrm{N}$ & Rank mean of IGF-1 & $\mathrm{df}$ & $\mathrm{p}$ \\
\hline 2 & 5 & 7.00 & 2 & 0.368 \\
4 & 5 & 8.50 & & \\
6 & 5 & 8.50 & & \\
\hline
\end{tabular}

Table 5 Comparison of IGF-1 level in disc in treated group at three different periods

\begin{tabular}{lcccc}
\hline Weeks & $\mathrm{N}$ & Rank mean of IGF-1 & $\mathrm{df}$ & $\mathrm{p}$ \\
\hline 2 & 5 & 6.50 & 2 & 0.727 \\
4 & 5 & 8.00 & & \\
6 & 5 & 9.50 & & \\
\hline
\end{tabular}

Adult male Sprague-Dawley (SD) rats (weight: 180-200 g) were raised in a clean environment in the Animal Experimental Center of Hebei Medical University.

\section{Preparation for the diabetic rat model}

$S D$ rats in the treated group were injected intraperitoneally with $30 \mathrm{mg} / \mathrm{kg}$ STZ solution (Cayman, Ann Arbor, MI) dissolved in a citric acid buffer (Solarbio, Beijing) three times every three days to maintain hyperglycemia and to induce diabetic rats. Fasting blood glucose was measured by a rapid glucose meter (Rightest GM550, Hua guang sheng ji, Taiwan). If blood glucose was $\geq 200 \mathrm{mg} / \mathrm{dL}$, the rat could be confirmed as a diabetic rat. Pre-test results showed that the established success rate of the diabetic rat model was $80 \%$. Finally, 15 diabetic SD rats were included in the treated group. Citric acid buffer was injected intraperitoneally in the control group, and 15 SD rats were included in the control group.

\section{Treatment for specimens}

Five rats were taken from each group in the second, fourth, and sixth week, respectively. The rats were killed by $1 \%$ pentobarbital sodium through intraperitoneal injection. Fresh lumbar disc tissues were obtained, weighted, and then rinsed with 0.02 mol/L of phosphate-buffered saline (PBS). Tissue blocks less than $0.5 \mathrm{~cm} \times 0.5 \mathrm{~cm} \times 0.1 \mathrm{~cm}$ were fixed with $4 \%$ paraformaldehyde, and ethanol was used for gradient elution. Paraffin tissue blocks were then embedded by a copper mold.

\section{HE staining}

The paraffin section was dewaxed by xylene, and gradient ethanol hydration was performed. The section was observed under a microscope after 5 min hematoxylin staining, washing with tap water, differentiating in a differentiation solution for $30 \mathrm{~s}$, soaking in tap water for $15 \mathrm{~min}, 2 \mathrm{~min}$ eosin staining, washing with tap water, gradient elution using ethanol,

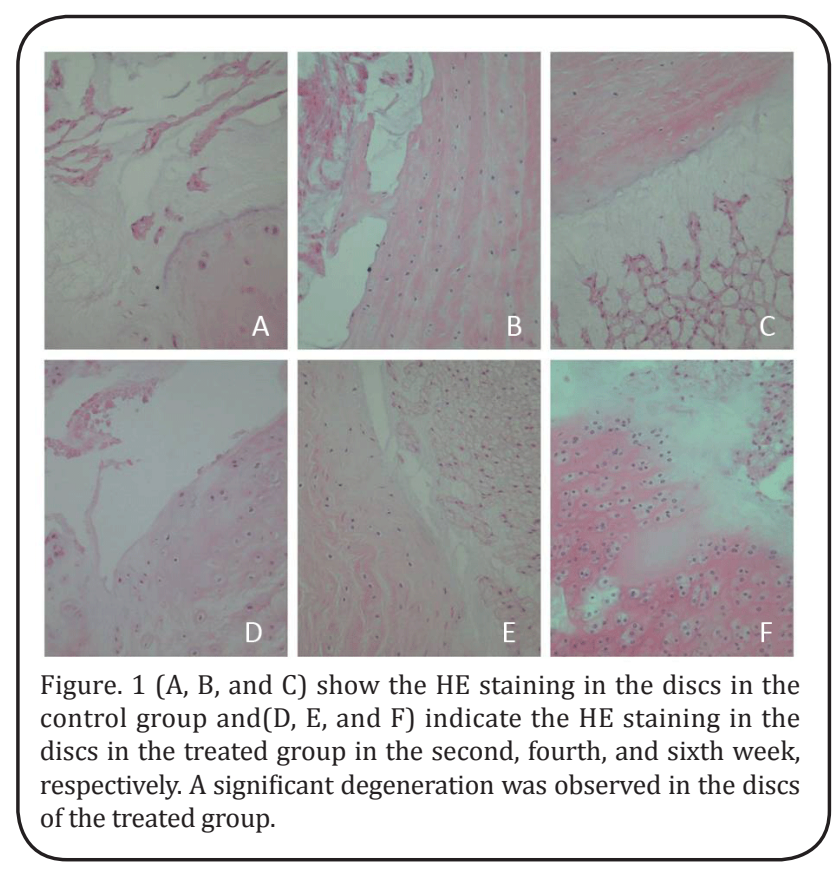




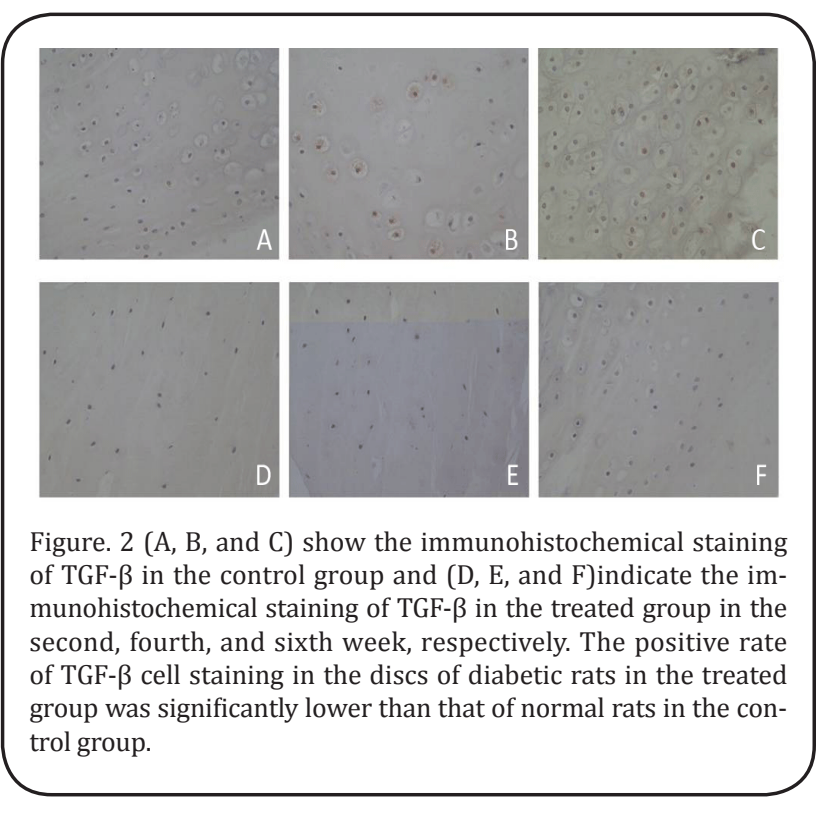

transparency with xylene, and mounting with neutral resins.

\section{Immunohistochemical method}

The paraffin section was cut into 5 um tissue sections and attached to slides treated by polylysine. The tissue sections were baked for $4.5 \mathrm{~h}$ at $60^{\circ} \mathrm{C}$. The wax was streaked after dewaxing and placed in PBS. The primary antibody was dropped onto the section at 4 ${ }^{\circ} \mathrm{C}$ overnight. After $10-20 \mathrm{~min}$ at room temperature, the sections were placed in PBS. The biotinylated second antibody was dropped onto the section at $37{ }^{\circ} \mathrm{C}$ for $20 \mathrm{~min}$. The sections were placed in PBS. Finally, streptavidin horseradish peroxidase conjugate was dropped onto the section at $37^{\circ} \mathrm{C}$ for $20 \mathrm{~min}$. The sections were placed in PBS. Diaminobenzidine (DAB) color was used. The nucleus was redyed by hematoxylin. The section was observed under a microscope after gradient elution using ethanol, transparency with xylene, and mounting with neutral resins.

\section{Scoring criteria}

The images were analyzed by Image-Pro Plus v 5.1. The histomorphology photos were collected by a microscope. The five strongest positive expressions of a visual field were calculated and assessed by cytoplasm staining and a positive cell rate. No coloring, light yellow, brownish yellow, and chocolate brown in the cytoplasm scored $0,1,2$, and 3 points, respectively. The positive cells $<5 \%$, between $5 \%$ and $25 \%$, between $26 \%$ and $50 \%$, between $51 \%$ and $75 \%$, and $>75 \%$ scored $0,1,2,3$, and 4 points, respectively. The total points of the two scores $<2$ points, between 2 and 3 points, between 3 and 4 points, between 4 and 5 points, and between 5 and 6 points indicated negative, weakly positive, positive, and strongly positive, respectively [8].

\section{Statistical analysis}

The data of the score were analyzed by the KruskalWallis $\mathrm{H}$ test. $\mathrm{P}$ value $<0.05$ was considered statistically significant.

\section{RESUITS}

\section{Blood glucose in rats}

The blood glucose concentrations in diabetic rats after treatment with STZ solution were significantly higher than those in the control group $(\mathrm{p}<0.05)$ (Table 1$)$.

\section{Disc degeneration in diabetic rats}

In the treated group, HE staining showed different degrees of degeneration changes in the discs of diabetic rats. Notochordal cells decreased, collagen fiber hyperplasia increased, and different degrees of endplate calcification were observed. Chondrocyte occurred and annulus fibrosus was arranged meanderingly. The nucleus pulposus matrix decreased, was arranged in a disorderly manner, and had different degrees of shrinkage. The nucleus pulposus and annulus fibrosus bulged outward. A part of the nucleus pulposus cells was arranged in clusters, which is a sign of disc degeneration. In control group, the structure of disc was normal. The endplate consisted of a layer of hyaline cartilage, and the annulus fibrosus had a wellregulated layered structure. The collagen bundles were regularly arranged and mainly consisted of fibroblasts. The boundary between the nucleus pulposus and annulus fibrosus was clear and full of notochordal cells (Figure. 1).

\section{Changes in the TGF- $\beta$ and IGF-1 level}

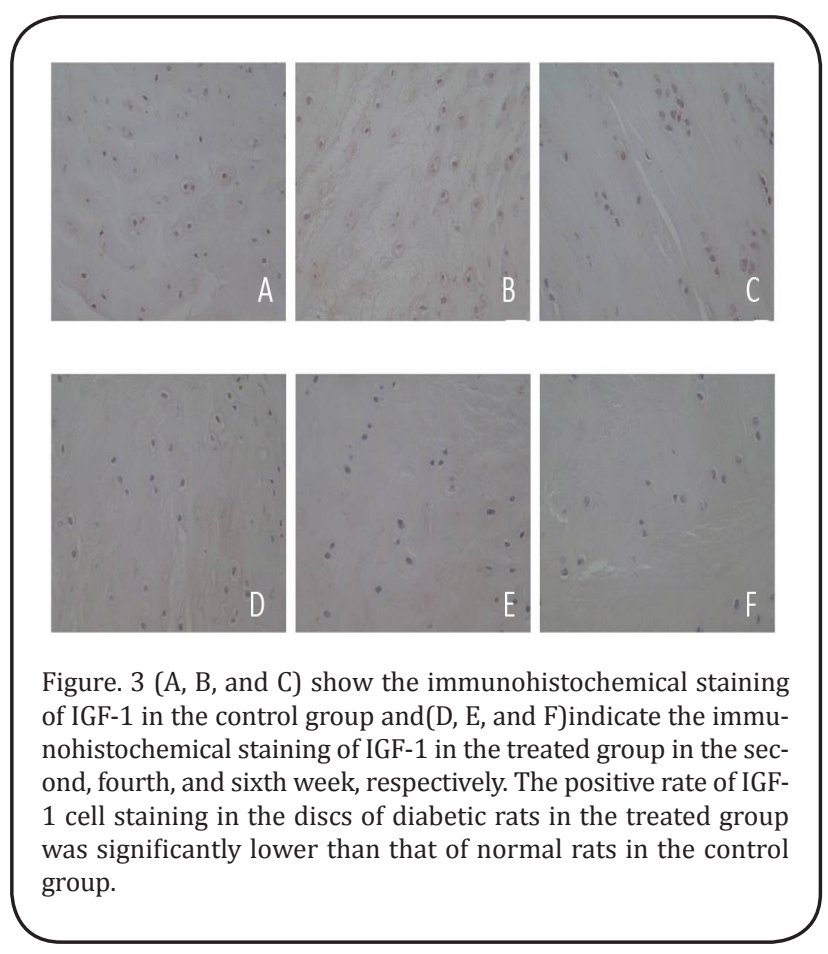


Table 6 Comparison of TGF- $\beta$ level in disc between control group and treated group

\begin{tabular}{|c|c|c|c|c|c|c|c|c|c|}
\hline \multirow[t]{2}{*}{ Group } & \multicolumn{5}{|c|}{ TGF- $\beta$ level } & \multirow{2}{*}{$\begin{array}{l}\text { Rank } \\
\text { mean }\end{array}$} & \multirow{2}{*}{$\begin{array}{l}\text { Rank } \\
\text { sum }\end{array}$} & \multirow[t]{2}{*}{ Z } & \multirow[t]{2}{*}{$\mathrm{p}$} \\
\hline & - & \pm & + & ++ & Total & & & & \\
\hline Control group & 0 & 0 & 1 & 14 & 15 & 23.00 & 345.00 & 5.109 & $0.000^{\star}$ \\
\hline Treated group & 3 & 12 & 0 & 0 & 15 & 8.00 & 120.00 & & \\
\hline Total & 3 & 12 & 1 & 14 & 30 & - & - & - & - \\
\hline
\end{tabular}

Table 7 Comparison of IGF-1 level in disc between control group and treated group

\begin{tabular}{|c|c|c|c|c|c|c|c|c|c|}
\hline \multirow[t]{2}{*}{ Group } & \multicolumn{5}{|c|}{ IGF-1 level } & \multirow{2}{*}{$\begin{array}{l}\text { Rank } \\
\text { mean }\end{array}$} & \multirow{2}{*}{$\begin{array}{r}\text { Rank } \\
\text { sum }\end{array}$} & \multirow[t]{2}{*}{$\mathrm{Z}$} & \multirow[t]{2}{*}{$\mathrm{p}$} \\
\hline & - & \pm & + & ++ & Total & & & & \\
\hline Control group & 0 & 0 & 1 & 14 & 15 & 23.00 & 345.00 & 5.068 & $0.000^{\star}$ \\
\hline Treated group & 4 & 11 & 0 & 0 & 15 & 8.00 & 120.00 & & \\
\hline Total & 4 & 11 & 1 & 14 & 30 & - & - & - & - \\
\hline
\end{tabular}

No significant difference was found between the TGF- $\beta$ and IGF-1 levels in the control group in three different periods $(\mathrm{p}>0.05)$ (Tables 2 and 4$)$. The situation in the treated group was the same (Tables 3 and 5).

The positive rate of the TGF- $\beta$ and IGF-1 cell staining in the discs of diabetic rats in the treated group was significantly lower than that of the normal rats in the control group in the three different periods $(\mathrm{p}<0.05)$ (Figure. 2 and 3; Tables 6 and 7).

\section{DISCUSSION}

Diabetes can negatively affect disc tissues ${ }^{[9]}$. In microvascular count research, Chen ${ }^{[10]}$ found that morphological damage in the microvessel in diabetic rats was more severe than that in normal rats, and microvascular count had a negative correlation with the apoptosis rate of nucleus pulposus cells. Hyperglycemia can increase the apoptosis rate of nucleus pulposus cells and shorten the time of disc degeneration. The accumulation of advanced glycation end (AGE) products increases, thus promoting the formation of oxygen free radicals and damaged mitochondria through oxidative stress. The AGE binding its receptor can increase the secretion of TGF- $\beta$ and IGF-1, which reduce the number of bone cells and accelerate disc degeneration.

According to the analysis of TGF- $\beta$ and IGF-1 levels in the discs in the control and treated groups, the TGF- $\beta$ and IGF- 1 levels in diabetic rats significantly decreased. Thus, the change in TGF- $\beta$ and IGF- 1 levels in the disc was one of the causes of disc degeneration. TGF- $\beta$ is a type of dimeric polypeptide, and it promotes the synthesis of cartilage matrix through a non-Smaddependent signaling pathway ${ }^{[11]}$. TGF- $\beta$ inhibits the expression and secretion of extracellular matrixdegrading enzymes to decrease the degradation of the extracellular matrix. Extracellular matrix-degrading enzymes in the disc affected by TGF- $\beta$ mainly include matrix metalloproteinase (MMPs), aggrecanase, and tissue inhibitor of metalloproteinase. MMPs can hydrolyze proteoglycan, collagen, and fibronectin. Yurube ${ }^{[12]}$ proved that MMP expression is closely related to disc degeneration. Aggrecanase can act on proteoglycan and degrade the extracellular matrix. The decrease in TGF- $\beta$ weakened the inhibition of extracellular matrix-degrading enzymes, thus resulting in the degradation of the extracellular matrix, accelerated the hydrolysis of collagen in the disc, reduced the osmotic pressure of the extracellular matrix and water content of the nucleus pulposus, and affected the structure and morphology of the disc.

IGF-1 is an active polypeptide that can inhibit cartilage degeneration, promote chondrocyte proliferation, and maintain the stability of cartilage collagen in the disc through the Extracellular Signal-regulated Kinase/Mitogen-activated Protein Kinase (ERK/ MAPK) signaling pathway ${ }^{[13]}$. Type II collagen is the main expression product in the cartilage endplate extracellular matrix in the disc ${ }^{[14]}$. If the amount of expression is reduced, disc degeneration occurs. IGF-1 can stimulate chondrocyte to synthesize the substrate-specific type II collagen, increase the activity of glycosaminoglycan, polyenzyme, and alkaline phosphatase of osteoblasts, protect the disc tissue, and prevent degeneration and aging. IGF-1 has a key role in the development and homeostasis in the disc ${ }^{[15]}$. In one study, exogenous IGF-1 was added in nucleus pulposus cells cultured in vitro, and proliferation was promoted in a dose-dependent manner ${ }^{[16]}$. IGF-1 can also stimulate nucleus pulposus cells to secrete the extracellular matrix and to inhibit the apoptosis of disc cells, as proved by Gruber ${ }^{[17]}$.

In conclusion, TGF- $\beta$ and IGF- 1 had a positive effect on the disc by affecting the activity of chondrocyte in the disc and the synthesis and degradation of the extracellular matrix. The decrease in TGF- $\beta$ and IGF-1 in the disc under a diabetic state is one of the reasons for the occurrence of degenerative changes in the disc among diabetics. Aside from preventing microangiopathy in diabetics and the positive control of blood sugar, the local injection of TGF- $\beta$ and IGF- 
1 can regulate the synthesis and degradation of the extracellular matrix and reduce the apoptosis of chondrocyte in the disc to prevent and treat degenerative disc changes among diabetics.

\section{CONFLIOT OF INTEREST}

All authors declare that there is no conflict of interest.

\section{REFERENCES}

1. Guariguata, L., Whiting, D., Weil, C., and Unwin, N. (2011) The International Diabetes Federation diabetes atlas methodology for estimating global and national prevalence of diabetes in adults. Diabetes Research \& Clinical Practice 94, 322-332

2. Ziv, I., Moskowitz, R. W., Kraise, I., Adler, J. H., and Maroudas, A. (1992) Physicochemical properties of the aging and diabetic sand rat intervertebral disc. Journal of Orthopaedic Research Official Publication of the Orthopaedic Research Society 10, 205-210

3. Zhao, C. Q., Zhang, Y. H., Jiang, S. D., Jiang, L. S., and Dai, L. Y. (2009) Both endoplasmic reticulum and mitochondria are involved in disc cell apoptosis and intervertebral disc degeneration in rats. Age 32, 161-177

4. Howard, B. V., Rodriguez, B. L., Bennett, P. H., Harris, M. I., Hamman, R., Kuller, L. H., Pearson, T. A., and Wylie-Rosett, J. (2002) Prevention Conference VI: Diabetes and Cardiovascular disease: Writing Group I: epidemiology. Circulation 105, e132-137

5. Huang, C. Y. (2011) Effect of endplate calcification and mechanical deformation on the distribution of glucose in intervertebral disc: a 3D finite element study. Computer Methods in Biomechanics \& Biomedical Engineering 14, 195-204

6. Won, H. Y., Park, J. B., Park, E. Y., and Riew, K. D. (2009) Effect of hyperglycemia on apoptosis of notochordal cells and intervertebral disc degeneration in diabetic rats. Journal of Neurosurgery Spine 11, 741-748

7. 7.Z, S., G, Q., Y, Z., S, G., Y, W., J, Z., J, S., and H, Z. (2014) The effect of unfused segments in coronal balance reconstitution after posterior selective thoracolumbar/lumbar fusion in adolescent idiopathic scoliosis. Spine 39, 2042-2048

8. Rhodes, A., Jasani, B., Balaton, A. J., and Miller, K. D. (2000) Immunohistochemical demonstration of oestrogen and progesterone receptors: correlation of standards achieved on in house tumours with that achieved on external quality assessment material in over 150 laboratories from 26 countries. Journal of Clinical Pathology 53, 292-
301

9. Gu, D., Reynolds, K., Duan, X., Xin, X., Chen, J., Wu, X., Mo, J., Whelton, P. K., and He, J. (2003) Prevalence of diabetes and impaired fasting glucose in the Chinese adult population: International Collaborative Study of Cardiovascular Disease in Asia (InterASIA). Diabetologia 46, 1190-1198

10. Chen, S., Liao, M., Li, J., Peng, H., and Xiong, M. (2013) The correlation between microvessel pathological changes of the endplate and degeneration of the intervertebral disc in diabetic rats. Experimental and therapeutic medicine 5, 711-717

11. Wrana, J. L. (2013) Signaling by the TGF $\beta$ superfamily. Cold Spring Harbor Perspectives in Biology 5, a011197-a011197

12. Yurube, T., Takada, T., Suzuki, T., Kakutani, K., Maeno, K., Doita, M., Kurosaka, M., and Nishida, K. (2012) Rat tail static compression model mimics extracellular matrix metabolic imbalances of matrix metalloproteinases, aggrecanases, and tissue inhibitors of metalloproteinases in intervertebral disc degeneration. Arthritis Research \& Therapy 14, 1-14

13. Yamm ani, R. R., and Loeser, R. F. ( $\left.\begin{array}{llll}2 & 0 & 1 & 2\end{array}\right)$ Extracellular nicotinamide phosphoribosyltransferase (NAMPT/visfatin) inhibits insulin-like growth factor-1 signaling and proteoglycan synthesis in human articular chondrocytes. Arthritis Research \& Therapy 14, : R23.

14. Ahsan, R., Tajima, N., Chosa, E., Sugamata, M., Sumida, M., and Hamada, M. (2001) Biochemical and morphological changes in herniated human intervertebral disc. Journal of Orthopaedic Science Official Journal of the Japanese Orthopaedic Association 6, 510-518

15. Urano, T., Narusawa, K. I., Shiraki, M., Usui, T., Sasaki, N., Hosoi, T., Ouchi, Y., Nakamura, T., and Inoue, S. (2008) Association of a Single Nucleotide Polymorphism in the Insulin-Like Growth Factor-1 Receptor Gene With Spinal Disc Degeneration in Postmenopausal Japanese Women. Spine 33, 12561261

16. Pratsinis, H., and Kletsas, D. (2007) PDGF, bFGF and IGF-I stimulate the proliferation of intervertebral disc cells in vitro via the activation of the ERK and Akt signaling pathways. European Spine Journal 16, 1858-1866

17. Gruber, H. E., Norton, H. J., and Jr, H. E. (2000) Antiapoptotic effects of IGF-1 and PDGF on human intervertebral disc cells in vitro. Spine 25, 21532157 